\title{
A Condition for Classical Elastic Curves on Surface
}

\author{
Ayşe Altın
}

(Communicated by Yusuf YAYLI)

\begin{abstract}
In this paper, we consider two fixed points $p$ to $q$ on a Riemannian surface $M$ in 3-dimensional Euclidean space. We obtain a condition for classical elastic curves with in the family of all curves from $p$ to $q$ on $M$. We also prove that this condition can be expressed in terms of the curvature functions. The condition is realized for curves whose geodesic and normal curvature functions are both constant.
\end{abstract}

Keywords: Energy, Energy of a unit vector field, Elastica.

AMS Subject Classification (2010): Primary: 53A04, ; Secondary: 53C20.

\section{Introduction}

The problem of the elastic rod which was proposed by Bernoulli is one of the most classical topics in variational calculus. As regards Bernoulli's mathematical idealization, all kinds of elastic curves minimize the total squared curvature among curves of the same length and first order boundary data [1]. Like many of the problems explored by mathematicians of this era, the formal investigation of the elastica was motivated by a physical situation. The Euler-Bernoulli treatment of the elastica transformed a physics problem into one in mathematics. Examining the bending energy of a physical elastic rod was replaced by investigating the total squared curvature of a regular curve [2]. The total squared curvature functional has emerged as a useful quantity in the study of geodesics and the closed thin elastic rod is often used as a model for the DNA molecule [3].

One of the earliest approach on elastica yields prolific consequences on equilibrium of moments which constitute elemantary principle of statics. Further, it is seen that elastica gives a natural solution for the variotional problem which deal with the minimizing of bending energy of the elastic curve. Later, the equivalance between the motion of the simple pendulum and fundamental differential equation of elastica was investigated. Recently, numerical computation implemented on the elastica used to develop math- ematical spline theory [4].

The volume of unit vector fields has been studied by [5], [6], and [7] among other scientists. They define the volume of unit vector field $X$ as the volume of the submanifold of the unit tangent bundle defined by $X(M)$. In [8], the energy of a unit vector field on a Riemannian manifold $M$ is defined as the energy of the mapping $X: M \rightarrow T^{1} M$, where the unit tangent bundle $T^{1} M$ is equipped with the restriction of the Sasaki metric on $T M$. The general references [9] and [10] are the classical notation of curve and of surface theory. In [11] author calculated the energy of the Frenet vector fields in $R^{n}$, it was shown that the energy of the velocity vector field was $\mathcal{E}\left(V_{1}(s)\right)=\frac{1}{2} \int_{a}^{s} k_{1}^{2}(u) d u$.

In this paper, similar to $R^{n}$ space, we give a condition for the elastica by defining the surface curve to be elastic. Finally, we give an example to elastic curve on the cylinder.

Definition 1.1 Let $M$ be a surface in $R^{3}$. If $p$ is a point of $M$, then for each tangent vector $v$ to $M$ at $p$, let

$$
S_{p}(v)=-\nabla_{v} Z
$$

Received : 28-11-2017, Accepted : 19-02-2018 
where $Z$ is a unit normal vector field on a neighborhood of $p$ in $M . S_{p}$ is called the shape operator of $M$ at $p$ (derived from $Z$ ).

Definition 1.2 A regular curve $\alpha$ in $M \subset R^{3}$ is a principal curve (or line of curvature) provided that the velocity $\alpha^{\prime}$ of $\alpha$ always points in a principal direction.

Definition 1.3 A regular curve $\alpha$ in $M \subset R^{3}$ is a geodesic of $M$ provided its acceleration $\alpha^{\prime \prime}$ is always normal to $M$.

Definition 1.4 Let $\alpha$ be a unit-speed curve in $M \subset R^{3}$. Instead of the Frenet frame field on $\alpha$, consider the frame field $\{T, Y, Z\}$, where $T$ is the unit tangent of $\alpha, Z$ is the surface normal restricted to $\alpha$, and , $Y=Z \times T$ then the following relations are valid.

$$
\begin{array}{r}
T^{\prime}=K_{g} Y+K_{n} Z \\
Y^{\prime}=-K_{g} T+K_{t} Z \\
Z^{\prime}=-K_{n} T-K_{t} Y .
\end{array}
$$

where $K_{n}=<S(T), T>$ is the normal curvature $K_{n}(T)$ of in the $T$ direction, and $K_{t}=<S(T), Y>$ is the torsion function. The new function $K_{g}$ is called the geodesic curvature of $\alpha$.

Definition 1.5 Let $\alpha$ be a unit-speed curve in $M \subset R^{3}$.

$$
\begin{gathered}
\text { Then } \alpha \text { is geodesic } \Leftrightarrow K_{g}=0, \\
\text { then } \alpha \text { is asymptotic } \Leftrightarrow K_{n}=0 .
\end{gathered}
$$

Definition 1.6 A curve segment is the portion of a curve defined in a closed interval.

Definition 1.7 Let $V$ and $W$ be two vector fields on $M$, and $Z$ be a normal vector field on $M$. We may then decompose $D_{V} W$ as

$$
D_{V} W=\nabla_{V} W+I I(V, W) Z
$$

where $\nabla_{V} W$ and $I I(V, W)$ are the tangential component and the normal component of $D_{V} W$, respectively. This decomposition is known as the Gauss equation.

Definition 1.8 For $\eta_{1}, \eta_{2} \in T_{\xi}\left(T^{1} M\right)$ define

$$
g_{\mathcal{S}}\left(\eta_{1}, \eta_{2}\right)=<d \pi\left(\eta_{1}\right), d \pi\left(\eta_{2}\right)>+<K\left(\eta_{1}\right), K\left(\eta_{2}\right)>.
$$

This gives a Riemannian metric on $T M$. Recall that $g_{\mathcal{S}}$ is called the Sasaki metric. The metric $g_{s}$ makes the projection $\pi: T^{1} M \rightarrow M$ a Riemannian submersion [12] and [13].

Proposition 1.1 The connection map $K: T\left(T^{1} M\right) \rightarrow T^{1} M$ verifies the following conditions.

1) $\pi \circ K=\pi \circ d \pi$ and $\pi \circ K=\pi \circ \widetilde{\pi}$, where $\widetilde{\pi}: T\left(T^{1} M\right) \rightarrow T^{1} M$ is the tangent bundle projection.

2) For $\omega \in T_{x} M$ and a section $\xi: M \rightarrow T^{1} M$, we have

$$
K(d \xi(\omega))=\nabla_{\omega} \xi
$$

where $\nabla$ is the Levi-Civita covariant derivative [12].

Definition 1.9. The energy of a differentiable map $f:(M,<,>) \rightarrow(N, h)$ between Riemannian manifolds is given by

$$
\mathcal{E}(f)=\frac{1}{2} \int_{M}\left(\sum_{a=1}^{n} h\left(d f\left(e_{a}\right), d f\left(e_{a}\right)\right) v\right.
$$

where $v$ is the canonical volume form in $M$ and $\left\{e_{a}\right\}$ is a local basis of the tangent space (see $[8,14]$, for example). 
Let $C^{\infty}(M ; N)$ denote the space of all smooth maps from $M$ to $N$. A map $f: M \rightarrow N$ is said to be harmonic if it is an extremal (i.e., critical point) of the energy functional $E(. ; D): C^{\infty}(M ; N) \rightarrow R$ for any compact domain $\mathrm{D}$.

By a (smooth) variation of $f$ we mean a smooth map $f: M \times(-\epsilon, \epsilon) \rightarrow N,(x, t) \rightarrow f_{t}(x)(\epsilon>0)$ such that $f_{0}=f$. We can think of $\left\{f_{t}\right\}$ as a family of smooth mappings which depends 'smoothly' on a parameter $t \in(-\epsilon, \epsilon)$.

Definition 1.10. A smooth map $f:(M, g) \rightarrow(N, h)$ is said to be harmonic if

$$
\left.\frac{d}{d t} \mathcal{E}\left(f_{t} ; D\right)\right|_{t=0}=o
$$

where $E(f ; D)=\frac{1}{2} \int_{D}\left(\sum_{a=1}^{n} h\left(d f\left(e_{a}\right), d f\left(e_{a}\right)\right) v_{g}\right.$, for all compact domains $D$ and all smooth variations $f_{t}$ of $f$ supported in $D$ [15].

Definition 1.11 Let $\alpha$ be a regular curve defined on any fixed interval [a; b]. Elastica is defined for the curve $\alpha$ in $R^{n}$ over the each point on a fixed interval $[a ; b]$ as a minimizer of the bending energy:

$$
\mathcal{E}_{B}=\frac{1}{2} \int_{a}^{b} k_{1}^{2}(s) d s
$$

with some boundary conditions [15] and [16].

\section{Classical Elastic Curves on Surface}

Definition 2.1. A regular curve on surface is called elastica if the velocity vector field of the curve is harmonic.

Let $\varphi: U \subset R^{2} \rightarrow R^{3}, \varphi(U) \subset M, \varphi(U)=\left(\varphi_{1}(u, v), \varphi_{2}(u, v), \varphi_{3}(u, v)\right)$ and $\varphi(u, v)$ be a local parametrization of surface $M$ in $R^{3}$.

Theorem 2.1. Let $\alpha$ be unit speed curve on surface $M$ and $\alpha(a)=p, \alpha(b)=q$. If $\alpha$ is classical elastic curve, then the following equation is satisfied,

$$
\int_{a}^{b} \lambda(s)\left(K_{g}(s) K_{g}^{\prime}(s)+K_{n}(s) K_{n}^{\prime}(s)\right) d s=0
$$

where $K_{n}, K_{g}$ are the normal curvature function and the geodesic curvature function of $\alpha$ and $\lambda$ is the real-valued function on $[a, b]$.

Proof. Let $(I, \alpha)$ be a parametric pair for a unit speed curve $C$ on $\varphi(U) \subset M$ and $\alpha=\varphi \circ \gamma, \gamma=\left(\gamma_{1}, \gamma_{2}\right)$. Let $\{T, Y, Z\}$ be the Frenet frame field on $\alpha$ in $M$.

We define $\lambda$ and $v_{i}$ functions to get a fixed two points on the surface and a collection of curves passing through these two points.

There existe $\lambda:[a, b] \subset I \rightarrow R, \lambda(s)=(s-a)(b-s), \lambda(a)=0, \lambda(b)=0$ and $\lambda(s) \neq 0$ for all $s \in(a, b)$, of class $C^{2}$. Since $\left\{\varphi_{1}(\gamma(s)), \varphi_{2}(\gamma(s))\right\}$ is a local basis of the tangent space, where $\varphi_{1}, \varphi_{2}$ are first-order partial derivatives, we have

$$
\lambda(s) T(s)=\Sigma_{i=1}^{2} v_{i}(s) \varphi_{i}(\gamma(s)) ; \text { where } v_{i}:[a, b] \rightarrow R .
$$

Let the collection of curve be

$$
\alpha^{k}(s)=\varphi\left(\gamma_{1}(s)+k v_{1}(s), \gamma_{2}(s)+k v_{2}(s)\right)
$$

for $k=0, \quad \alpha^{0}(s)=\alpha(s)$ and

$$
\left(\varphi^{-1} \circ \alpha^{k}\right)(s)=\gamma^{k}(s)=\left(\gamma_{1}(s)+k v_{1}(s), \gamma_{2}(s)+k v_{2}(s)\right) .
$$

From (10) we get $\lambda(a) T(a)=\Sigma_{i=1}^{2} v_{i}(a) \varphi_{i}(\gamma(a))$. Since $\lambda(a)=0$ we have $v_{1}(a)=v_{2}(a)=0$ and

$$
\gamma^{k}(a)=\left(\gamma_{1}(a)+k v_{1}(a), \gamma_{2}(a)+k v_{2}(a)=\left(\gamma_{1}(a), \gamma_{2}(a)\right)=\gamma(a) .\right.
$$


Similarly we get $v_{1}(b)=v_{2}(b)=0$ and $\gamma^{k}(b)=\gamma(b)$. Using these results in (11) we obtain $\alpha^{k}(a)=\left(\varphi \circ \gamma^{k}\right)(a)=\alpha(a)=p$ and $\alpha^{k}(b)=\left(\varphi \circ \gamma^{k}\right)(b)=\alpha(b)=q$.

These results show that $\alpha^{k}$ is curve segment from $p$ to $q$ on $M$. Take this collection $\alpha^{k}(s)=\alpha(s, k)$ for all curve. The expression for energy of velocity vector field $T_{k}$ of $\alpha^{k}$ from $p$ to $q$ on $M$ becomes $\mathcal{E}\left(T_{k}\right)$.

On the other hand, let $T C_{k}$ be the tangent bundle. So we have $T_{k}: C_{k} \rightarrow T C_{k}=\bigcup_{s \in I} T_{\alpha^{k}(s)} C_{k}$., where $C_{k}=\alpha^{k}(I)$ and $T_{\alpha^{k}(s)} C_{k}$ denotes generated by $T_{k}$. Let $\pi: T C_{k} \rightarrow C_{k}$ be the bundle projection. By using equation (7) we obtain that the energy of $T_{k}$ is

$$
\mathcal{E}\left(T_{k}\right)=\frac{1}{2} \int_{a}^{b} g_{\mathcal{S}}\left(d T _ { k } \left(T_{k}(\alpha(s, k)), d T_{k}\left(T_{k}(\alpha(s, k))\right) d s\right.\right.
$$

where $d s$ is the element of arc length. From (6) we have

$$
g_{\mathcal{S}}\left(d T_{k}\left(T_{k}\right), d T_{k}\left(T_{k}\right)\right)=<d \pi\left(d T_{k}\left(T_{k}\right)\right), d \pi\left(d T_{k}\left(T_{k}\right)\right)>+<K\left(d T_{k}\left(T_{k}\right)\right), K\left(d T_{k}\left(T_{k}\right)\right)>.
$$

Since $T_{k}$ is a section we have $d(\pi) \circ d\left(T_{k}\right)=d\left(\pi \circ T_{k}\right)=d\left(i d_{C_{k}}\right)=i d_{T C_{k}}$ we also have by Proposition 1.1 that $K\left(d T_{k}\left(T_{k}\right)\right)=\nabla_{T_{k}} T_{k}=T_{k}^{\prime}$, then

$$
g_{\mathcal{S}}\left(d T_{k}(T), d T_{k}(T)\right)=<T_{k}, T_{k}>+<T_{k}^{\prime}, T_{k}^{\prime}>.
$$

Using these results in (12) we get

$$
\mathcal{E}\left(T_{k}\right)=\frac{1}{2} \int_{a}^{b}\left(<T_{k}, T_{k}>+<T_{k}^{\prime}, T_{k}^{\prime}>\right) d s
$$

where $T_{k}=\frac{1}{w(s, k)} \frac{\partial \alpha}{\partial s}(s, k) ; w(s, k)=\sqrt{<\frac{\partial \alpha}{\partial s}(s, k), \quad \frac{\partial \alpha}{\partial s}(s, k)>}, T_{k}^{\prime}=\frac{\partial T_{k}}{\partial s}$.

From (13) we obtain:

$$
\frac{\partial \mathcal{E}\left(T_{k}\right)}{\partial k}=\frac{1}{2}\left[\int _ { a } ^ { b } \frac { \partial } { \partial k } \left[\left(<T_{k}, T_{k}>+<\frac{\partial T_{k}}{\partial s}, \frac{\partial T_{k}}{\partial s}>\right] d s .\right.\right.
$$

Since $<T_{k}, T_{k}>=1$ we have $\frac{\partial}{\partial k}<T_{k}, T_{k}>=0$ and by using equation (1), we get

$$
\frac{\partial \mathcal{E}\left(T_{k}\right)}{\partial k}=\frac{1}{2}\left[\int_{a}^{b} \frac{\partial}{\partial k}<\frac{\partial T_{k}}{\partial s}, \frac{\partial T_{k}}{\partial s}>\right] d s=\frac{1}{2} \int_{a}^{b} \frac{\partial}{\partial k}\left(K_{g}^{2}+K_{n}^{2}\right) d s .
$$

Since $\alpha$ is classical elastic curve, by definition 2.1 and (8) we have

$$
{\frac{\partial \mathcal{E}\left(T_{k}\right)}{\partial k}}_{\mid k=0}=\left(\frac{1}{2} \int_{a}^{b} \frac{\partial}{\partial k}\left(K_{g}^{2}+K_{n}^{2}\right) d s\right)_{\mid k=0}=0 .
$$

from (14) we have

$$
\frac{\partial \mathcal{E}\left(T_{k}\right)}{\partial k}=\frac{1}{2} \int_{a}^{b} \frac{\partial}{\partial k}<\frac{\partial T_{k}}{\partial s}, \frac{\partial T_{k}}{\partial s}>d s=\int_{a}^{b}<\frac{\partial^{2} T_{k}}{\partial s \partial k}, \frac{\partial T_{k}}{\partial s}>d s
$$

We can write

$$
\frac{\partial}{\partial s}<\frac{\partial T_{k}}{\partial k}, \frac{\partial T_{k}}{\partial s}>=<\frac{\partial^{2} T_{k}}{\partial s \partial k}, \frac{\partial T_{k}}{\partial s}>+<\frac{\partial T_{k}}{\partial k}, \frac{\partial^{2} T_{k}}{\partial s^{2}}>
$$

Thus, we can deduce,

$$
<\frac{\partial^{2} T_{k}}{\partial s \partial k}, \frac{\partial T_{k}}{\partial s}>=\frac{\partial}{\partial s}<\frac{\partial T_{k}}{\partial k}, \frac{\partial T_{k}}{\partial s}>-<\frac{\partial T_{k}}{\partial k}, \frac{\partial^{2} T_{k}}{\partial s^{2}}>
$$


Substituting (17) in (16), for, $k=0$,

$$
{\frac{\partial \mathcal{E}\left(T_{k}\right)}{\partial k}}_{\mid k=0}=\int_{a}^{b}\left[\frac{\partial}{\partial s}<\frac{\partial T_{k}}{\partial k}(s, 0), \frac{\partial T_{k}}{\partial s}(s, 0)>-<\frac{\partial T_{k}}{\partial k}(s, 0), \frac{\partial^{2} T_{k}}{\partial s^{2}}(s, 0)>\right] d s
$$

and

$$
{\frac{\partial \mathcal{E}\left(T_{k}\right)}{\partial k}}_{\mid k=0}=<\frac{\partial T_{k}}{\partial k}(s, 0), \frac{\partial T_{k}}{\partial s}(s, 0)>\left.\right|_{a} ^{b}-\int_{a}^{b}<\frac{\partial T_{k}}{\partial k}(s, 0), \frac{\partial^{2} T_{k}}{\partial s^{2}}(s, 0)>d s .
$$

From (10), (11) we obtain,

$$
\frac{\partial \alpha}{\partial k}(s, k)_{\mid k=0}=\lambda(s) T(s)
$$

and

$$
\frac{\partial \alpha}{\partial s}(s, 0)=\alpha^{\prime}(s)=T(s)=T_{k}(s .0) .
$$

Now we calculate the partial derivatives of (20) with respect to s and k; using (1), we get

$$
\frac{\partial T_{k}}{\partial s}(s, 0)=\frac{\partial^{2} \alpha}{\partial s^{2}}(s, 0)=\alpha^{\prime \prime}(s)=T^{\prime}(s)=K_{g}(s) Y(s)+K_{n}(s) Z(s)
$$

and

$$
\frac{\partial T_{k}}{\partial k}(s, k)=\frac{\partial^{2} \alpha}{\partial s \partial k}(s, k)=\frac{\partial^{2} \alpha}{\partial k \partial s}(s, k)
$$

So, (19) gives us that

$$
\frac{\partial T}{\partial k}(s, k)_{\left.\right|_{k=0}}=\frac{\partial T}{\partial k}(s, 0)=\lambda^{\prime}(s) T(s)+\lambda(s) K_{g}(s) Y(s)+\lambda(s) K_{n}(s) Z(s) .
$$

Therefore, (21) and (22) gives us that

$$
<\frac{\partial T_{k}}{\partial k}(s, 0), \frac{\partial T_{k}}{\partial s}(s, 0)>=\lambda(s)\left(K_{g}^{2}(s)+K_{n}^{2}(s)\right) .
$$

Considering the candidate function $\lambda(a)=\lambda(b)=0$, we get:

$$
<\frac{\partial T_{k}}{\partial k}(s, 0), \frac{\partial T_{k}}{\partial s}(s, 0)>\left.\right|_{a} ^{b}=\lambda(b)\left(K_{g}^{2}(b)+K_{n}^{2}(b)\right)-\lambda(a)\left(K_{g}^{2}(b)+K_{n}^{2}(a)\right)=0 .
$$

Now we calculate the derivative (21) with respect to $s$,

$$
\frac{\partial^{2} T}{\partial s^{2}}(s, 0)=K_{g}^{\prime}(s) Y(s)+K_{g}(s) Y^{\prime}(s)+K_{n}^{\prime}(s) Z(s)+K_{n}(s) Z^{\prime}(s)
$$

By (2) and (3) we have

$$
\frac{\partial^{2} T}{\partial s^{2}}(s, 0)=-\left(K_{g}^{2}(s)+K_{n}^{2}(s)\right) T(s)+\left(K_{g}^{\prime}(s)-K_{n}(s) K_{t}(s)\right) Y(s)+\left(K_{g}(s) K_{t}(s)+K_{n}^{\prime}(s)\right) Z(s) .
$$

So (22) and (24) given us that

$$
<\frac{\partial T_{k}}{\partial k}(s, 0), \frac{\partial^{2} T_{k}}{\partial s^{2}}(s, 0)>=-\lambda^{\prime}(s)\left(K_{g}^{2}(s)+K_{n}^{2}(s)\right)+\lambda(s)\left(K_{g}(s) K_{g}^{\prime}(s)+K_{n}(s) K_{n}^{\prime}(s)\right)
$$


and

$$
<\frac{\partial T_{k}}{\partial k}(s, 0), \frac{\partial^{2} T_{k}}{\partial s^{2}}(s, 0)>=\left[-\lambda(s)\left(K_{g}^{2}(s)+K_{n}^{2}(s)\right)\right]^{\prime}+3 \lambda(s)\left(K_{g}(s) K_{g}^{\prime}(s)+K_{n}(s) K_{n}^{\prime}(s)\right)
$$

Using (23) and (25) in (18), yields that

$$
{\frac{\partial \mathcal{E}\left(T_{k}\right)}{\partial k}}_{\mid k=0}=-\int_{a}^{b}\left(\left[-\lambda(s)\left(K_{g}^{2}(s)+K_{n}^{2}(s)\right)\right]^{\prime}+3 \lambda(s)\left(K_{g}(s) K_{g}^{\prime}(s)+K_{n}(s) K_{n}^{\prime}(s)\right) d s\right.
$$

and

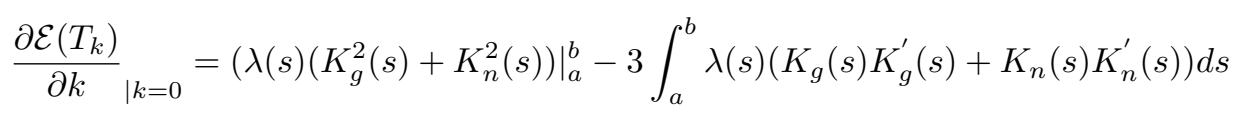

We are looking the candidate function $\lambda(a)=\lambda(b)=0$ which gives

$$
\left(\left.\lambda(s)\left(K_{g}^{2}(s)+K_{n}^{2}(s)\right)\right|_{a} ^{b}=0\right.
$$

from (15), we have

$$
\left.\frac{\partial \mathcal{E}\left(T_{k}\right)}{\partial k}\right|_{\mid k=0}=-3 \int_{a}^{b} \lambda(s)\left(K_{g}(s) K_{g}^{\prime}(s)+K_{n}(s) K_{n}^{\prime}(s)\right) d s=0 .
$$

This completes the proof of the theorem.

From equations (4)and (5), if $\alpha$ is both principal and asymptotic or the normal curvature function and the geodesic curvature function of $\alpha$ are constant, then is satisfy (9) equation.

Example 1. Let $\varphi:[-\pi, \pi] \times R \rightarrow R^{3}, \varphi(\theta, h)=(\cos \theta, \sin \theta, h$,$) and \beta(s)=(\cos (\cos s), \sin (\operatorname{coss}), \operatorname{sins}) ; \beta(-\pi)=$ $p, \beta(\pi)=q$. If we can choose $\lambda:[-\pi, \pi] \rightarrow R, \lambda(s)=\pi^{2}-s^{2}$ then $\lambda(-\pi)=0 \quad \lambda(\pi)=0$ and $\lambda(s) \neq 0$ for all $s \in(-\pi, \pi)$. We calculate;

$$
\begin{gathered}
T(s)=(\operatorname{sins} \cdot \sin (\cos s),-\operatorname{sins} \cdot \cos (\cos s), C o s s), \\
Z(s)=(\cos (\cos s), \sin (\cos s), 0), \\
Y(s)=(\operatorname{coss} \cdot \sin (\cos s),-\operatorname{coss} \cdot \cos (\cos s),-\sin s), \\
K_{g}(s)=1, K_{n}(s)=-\sin ^{2} s
\end{gathered}
$$

and

$$
{\frac{\partial \mathcal{E}\left(T_{k}\right)}{\partial k}}_{\mid k=0}=6 \int_{-\pi}^{\pi}\left(\pi^{2}-s^{2}\right) \sin ^{2} \text { s.sins.coss. } d s=0 .
$$

Thus $\beta$ is elastica on the cylinder, Figure 1.

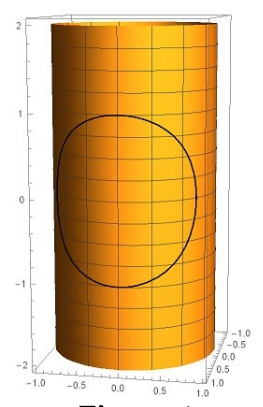

Figure 1 


\section{References}

[1] M. Barros, A. Ferrndez, P. Lucas and M. A. Meroo, "Willmore Tori and Willmore-Chen Submanifolds in Pseudo-Riemannian Spaces", Journal of Geometry and Physics, Vol. 28, pp. 45-66, 1998.

[2] D. H. Steinberg, "Elastic Curves in Hyperbolic Space", Doctoral Thesis, Case Western Reserve University, UMI Microform 9607925, 72p, 1995.

[3] R. Huang, "A Note on the p-elastica in a Constant Sectional Curvature Manifold", Journal of Geometry and Physics, Vol. 49, pp. 343-349, 2004.

[4] A. E. H. Love," "A treatise on the mathematical theory of elasticity", Cambridge University Press, 2013.

[5] H. Gluck, W. Ziller, "On the volume of the unit vector fields on the three sphere", Comment Math. Helv, Vol. 61, pp. 177-192, 1986.

[6] D. L. Johnson, "Volume of flows", Proc. Amer. Math. Soc, Vol. 104, pp. 923-932, 1988.

[7] A. Higuchi, B. S. Kay, C. M. Wood, "The energy of unit vector fields on the 3-sphere". Journal of Geometry and Physics, Vol. 37, pp. 137-155, 2001.

[8] C. M. Wood, "On the Energy of a Unit Vector Field", Geometrae Dedicata, Vol. 64, pp. 319-330 1997.

[9] B. O’Neill, "Elementary Diffrential Geometry", Academic Press Inc., 1966.

[10] D. J. Struik, "Differential Geometry", Reading MA: Addison-Wesley, 1961.

[11] A. Altın, "On the Energy of Frenet Vectors Fields in $R^{n "}$, Cogent Mathematics, 2017.

[12] P. A. Chacón, A. M. Naveira, J. M. Weston, "On the Energy of Distributions, with Application to the Quarternionic Hopf Fibration", Monatshefte fûr Mathematik, Vol. 133, pp. 281-294, 2001.

[13] T. Sakai, "Riemannian Geometry", American Mathematical Society, 1996.

[14] P. M. Chacón, A. M. Naveira, "Corrected Energy of Distributions on Riemannian Manifold", Osaka Journal Mathematics, Vol 41, pp. 97-105, 2004.

[15] L. Euler, "Additamentum de curvis elasticis. In Methodus Inveniendi Lineas Curvas Maximi Minimive Probprietate Gaudentes". Lausanne, 1744.

[16] P. Baird, J. C. Wood, "Harmonic Morphisms Between Riemannian Manifold", Clarendos press, Oxford, 2003.

AYŞE ALTIN

AdDress: Hacettepe University,

Faculty of Science, Department of Mathematics

06550 Ankara, Turkey

E-MAIL: ayse@hacettepe.edu.tr

ORCID ID: $0000-0002-9470-5196$ 